\title{
Phase-Amplitude Coupling between EEG and EDA while experiencing multimedia content
}

\author{
Eleni Kroupi \\ Multimedia Signal \\ Processing Group (MMSPG) \\ École Polytechnique \\ Fédérale de Lausanne
}

(EPFL)

\author{
Jean-Marc Vesin \\ Applied Signal \\ Processing Group (ASPG) \\ École Polytechnique \\ Fédérale de Lausanne
}

(EPFL)

\author{
Touradj Ebrahimi \\ Multimedia Signal \\ Processing Group (MMSPG) \\ École Polytechnique \\ Fédérale de Lausanne
}

(EPFL)

\begin{abstract}
Emotion is a dynamic process that affects social relationships and influences the mechanisms of rational thinking and decision making. The influence of emotion on reasoning has gained a lot of attention in computer science. One approach to incorporating emotions into computers is by extracting features from various physiological signals and inferring the corresponding emotions or emotional dimensions. However, physiological processes are dynamic rather than static, and physiological signals mainly interact with each other during emotional processes rather than act individually. This study suggests that dynamical interdependencies of various signals that emanate from either the peripheral nervous system or the central nervous system might be involved in the emotional experience. Specifically, EEG (electroencephalography) signals captured from the temporal lobe are coupled with a narrow-band skin conductance response (SCR) while subjects are watching music clips, indicating that signals from the auditory cortex interact with SCR during this experience.
\end{abstract}

Index Terms-EEG, EDA, phase-amplitude coupling

\section{INTRODUCTION}

Emotion is a dynamic adaptive mechanism that involves various processes such as subjective experience and neurochemical activity. Moreover, emotions affect and determine social relationships and interactions, and influence the mechanisms of rational thinking and decision making [1]. For instance, patients who have normal intelligence scores but lack certain emotions act irrationally and are unable to make proper decisions [1]. Obviously, without emotions humans would need a huge amount of time to compute the gain of every response option and act according to the one that gives the highest gain for a certain situation [1]. Thus, emotions do not impair rationality as people used to believe, but they help, instead, to interact and make decisions properly.

The influence of emotion on decision making has gained a lot of interest and attention in computer science. By modeling emotion, researchers endeavor to add to machines an affective functionality that will make them more sensitive to humans and more efficient. For instance, adding emotion to computers can ease the selection of content from the vast amount of multimedia content available in the network. More specifically, users may feel frustrated and confused trying to find and select multimedia content that they are in the mood of experiencing at a certain moment. The process would be much faster and easier if computers were able to implicitly understand users' current needs, emotions and moods and take actions accordingly.

Much progress has been made in implicit emotion assessment from multimedia content. Typically, implicit emotion assessment is carried out through analysis of human expressions and physiological responses. Human expressions comprise verbal and non-verbal cues that can be processed using speech, gesture and face recognition. Physiological responses originate from the central nervous system (CNS) and the peripheral nervous system (PNS). Various PNS and CNS features are associated with emotions. For instance, heart rate increases when experiencing emotions such as anger, anxiety, surprise and happiness, while respiration rate as well as skin conductance response seem to increase with anger, anxiety, disgust, fear and amusement [2].

Among peripheral responses, electrodermal activity (EDA) is particularly important for emotion assessment. EDA is related to the activity of eccrine sweat glands. It is controlled by the sympathetic branch of ANS which is related to various psychological processes. Every stimulus that elicits an emotion produces changes in EDA proportional to the arousing level of the emotion experienced [3]. Moreover, EDA has been shown to covary with activity in certain brain regions, such as the ventromedial prefrontal cortex and the orbitofrontal cortex during biofeedback arousing tasks [4].

Regarding brain responses related to emotions, frontal-lobe asymmetric activity is highly associated with approach and withdrawal emotional processes [5], and acts as a moderator and mediator of emotion [6]. One of the main tools of brain research is electroencephalography (EEG) which has been widely used in studies that investigate associations between emotions and brain activity. In particular, power spectral density in various EEG frequency bands, among other EEG features, is highly associated with pleasant and unpleasant emotions elicited by various stimuli [7], [8].

Typically, in emotion recognition from physiological signals, static features are extracted from the signals and are used in a classification or regression analysis scheme to infer specific emotions or emotional dimensions [9], [10], [7], [11], [12]. However, the dynamic interrelation of CNS and 
PNS patterns during emotional processes has received less attention, although there is evidence that brain activation is associated with peripheral and other visceral responses [1], and that synchronization phenomena of various subsystems occur during emotional processes [13]. Regarding the association between peripheral and brain responses, several studies [4], [14] have reported frontal lobe activation by EDA, during biofeedback arousing and relaxation tasks. Yet these studies applied linear correlation measures to estimate the interactions between brain activity and EDA, although both EEG and EDA are complex, with non-linear characteristics [15], [16]. Also, a very recent study [17] used phase synchronization to detect the interaction between heart rate and respiration during affective picture viewing. However, both heart rate and respiration emanate from PNS, thus this study provides no information about the interactions or coupling between brain activity and peripheral responses.

The present study investigates how various brain regions interact with EDA in a non-linear way while experiencing affective multimedia content, i.e., music clips. There is evidence that oscillations at different frequencies interact with each other in a way such that the phase of the low-frequency oscillations modulates the amplitude of the high frequency oscillations, a process called phase-amplitude coupling (PAC). So far, PAC has been investigated in various frequency subbands of electrophysiological signals, such as gamma amplitude modulation by theta phase, and seems to be involved in various processes such as cognitive function, attentional selection and memory demands. In this study we use phase-amplitude coupling [18] in a new framework, to investigate potential amplitude modulation of the high-frequency EEG signals of different brain regions by the phase of the low-frequency EDA signals while experiencing multimedia content.

This paper is organized as follows. Section II describes the dataset, the methods used in this study and the proposed approach. The results are presented in Section III, and the further discussion is detailed in Section IV. Finally, the conclusions are presented in Section V.

\section{MATERIALS, METHODS AND PROPOSED APPROACH}

\section{A. Dataset}

The data of 32 participants used in this study was obtained from the DEAP database [19]. The physiological signals were recorded using a Biosemi ActiveTwo system with 32 active $\mathrm{AgCl}$ electrodes, four electrooculogram (EOG) electrodes and two EDA sensors, at a sampling frequency of $512 \mathrm{~Hz}$. EEG, EOG and EDA were recorded while forty 1-min music clips (carefully selected) were presented to each participant in a random order, constituting forty single trials.

\section{B. Signal Preprocessing}

EEG, EOG and EDA were downsampled to $128 \mathrm{~Hz}$. EEG and EOG signals were filtered between 3-47 Hz using a 3rd order Butterworth filter. EOG artifacts were removed using
FastICA $^{1}$, and the signals were re-referenced to the common average. Only two temporal, two occipital, two central, two prefrontal and two parietal electrodes are used in this study, namely T7, T8, O1, O2, C3, C4, Fp1, Fp2, P3 and P4 respectively. Furthermore, EDA reflects a rapid, transient response called skin conductance response (SCR), as well as a slower, basal shift called skin conductance level (SCL). Due to the fact that SCR is widely used in psychological studies as an objective metric for emotional processing [1], only SCR is used in this study.

\section{Phase-Amplitude Synchronization algorithm}

There is evidence that electrophysiological signals at different frequencies can interact with each other. Phase-amplitude coupling represents a possible type of interaction, corresponding to modulation of the amplitude of high frequency oscillators by the phase of low frequency rhythms [18]. Let $x(t)$ be the high frequency oscillator (EEG signal), and $y(t)$ be the low frequency rhythm (SCR signal). The instantaneous phase of $y(t)$ denoted by $\phi_{y}(t)$ is extracted, as well as the instantaneous amplitude of $x(t)$ denoted by $A_{x}(t)$. The phase is binned, and the normalized mean amplitude is defined as:

$$
P(n)=\frac{\left\langle A_{x}\right\rangle \phi_{y}(n)}{\sum_{k=1}^{N}\left\langle A_{x}\right\rangle \phi_{y}(k)},
$$

where $\left\langle A_{x}\right\rangle \phi_{y}(n)$ is the mean amplitude at phase bin $n$, and $N$ is the number of bins [18]. The normalized amplitude $P$ has the same characteristics as a probability distribution so the strength of the phase-amplitude coupling can be estimated from the divergence of the phase-amplitude distribution from a uniform one [18]. For this purpose, an index based on Kullback-Leibler (KL) divergence is used, which is referred to as the Modulation Index (MI) in [18]. The KL divergence between two distributions $P$ and $Q$ is formed as follows:

$$
D_{\mathrm{KL}}(P, Q)=\sum_{j=1}^{N} P(j) \log \left[\frac{P(j)}{Q(j)}\right] .
$$

The KL divergence has the property that $D_{\mathrm{KL}}(P, Q) \geq 0$, and $D_{\mathrm{KL}}(P, Q)=0$ only if the two distributions are identical. When $Q$ is the uniform distribution, $Q=U$, the KL divergence becomes

$$
D_{\mathrm{KL}}(P, U)=\log (N)-H(P),
$$

where

$$
H(P)=-\sum_{j=1}^{N} P(j) \log [P(j)]
$$

is the Shannon entropy of distribution $P$. The MI is then defined as

$$
\mathrm{MI}=\frac{D_{\mathrm{KL}}(P, U)}{\log (N)} .
$$

As MI increases, the phase-amplitude coupling becomes stronger since $P$ gets further away from $U$.

\footnotetext{
${ }^{1}$ http://www.cis.hut.fi/projects/ica/fastica/
} 


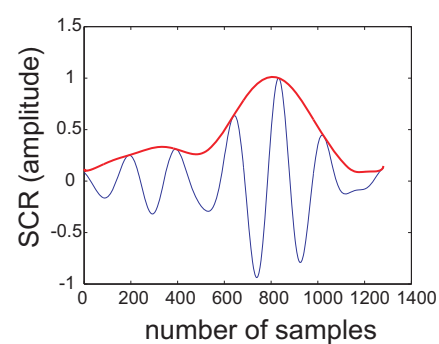

(a)

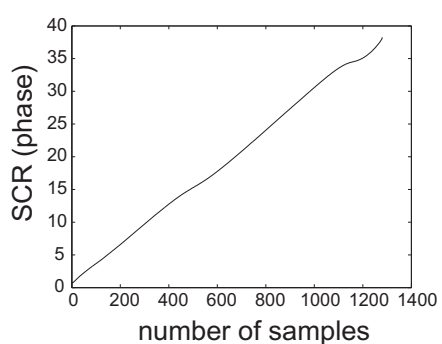

(b)

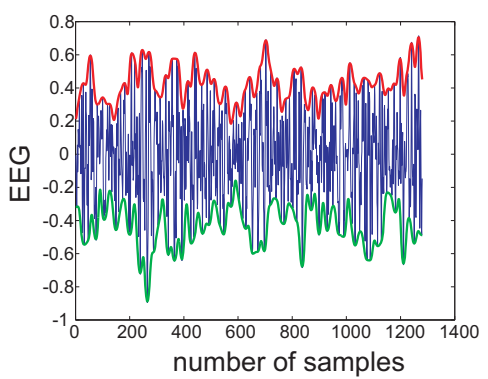

(c)

Fig. 1. (a) Envelope of an SCR segment estimated using HT. (b) Instantaneous phase (in radians) of the same SCR segment using HT. (c) Upper and lower envelope of an EEG segment estimated using a morphological operator.

\section{Instantaneous phase and amplitude estimation}

The unwrapped instantaneous phase $\phi_{y}(t)$ can be estimated by applying the analytical signal approach, according to which the instantaneous phase of an arbitrary signal $y(t)$ is defined as

$$
\begin{gathered}
\phi_{y}(t)=\arctan \frac{\tilde{y}(t)}{y(t)} . \\
\tilde{y}(t)=\frac{1}{\pi} \text { p.v. } \int_{-\infty}^{+\infty} \frac{y(\tau)}{t-\tau} d \tau
\end{gathered}
$$

is the Hilbert transform (HT) of the signal, and p.v. denotes the Cauchy principal value. In our case SCR is narrow-band (0.5-1 Hz), so HT can be applied to the SCR signals. The envelope and phase of an SCR segment estimated using HT are presented in Fig. 1(a-b). The fact that the envelope is captured accurately indicates that the phase is captured accurately as well.

However, since EEG signals are not narrow-band, the HT cannot be applied directly. Thus, the upper and lower envelopes of the EEG signals are estimated using a morphological operator (maxima and minima computed on a sliding window). The visible lack of symmetry between upper and lower envelopes (Fig. 1(c)) points indeed to the fact that HT cannot be used on these EEG signals. Only the upper envelope is used in this study.

\section{RESULTS}

\section{A. Coupling among various brain regions and SCR}

The synchronization between EEG and SCR was estimated in the following way; first, to consider the same range of

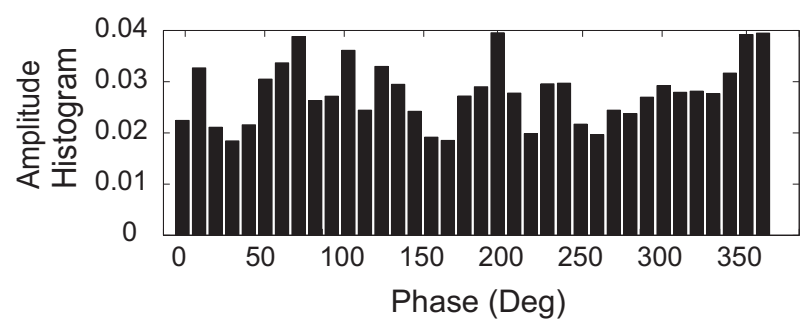

Fig. 2. Example of a Phase-Amplitude plot.

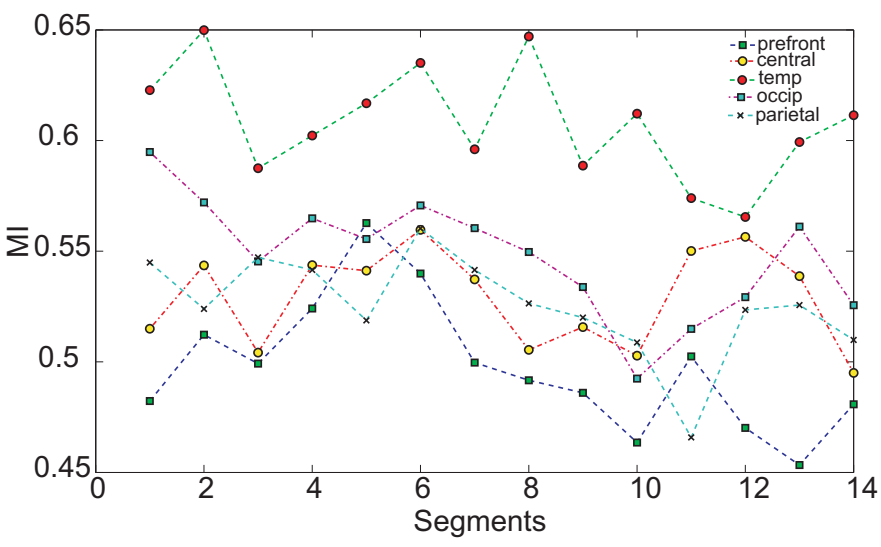

Fig. 3. MI index over time for a trial. The MI of the temporal lobe seems to be larger than any other MI.

values, each signal was normalized in the $[-1,1]$ range. Then each EEG signal, and the corresponding SCR signal were segmented using 10 -sec overlapping windows (60\% overlap) leading to 14 segments. Once the phase of each SCR segment was estimated using HT (Fig. 1(a-b)), as well as the envelope of the corresponding EEG segment (Fig. 1(c)), then the phaseamplitude plot was constructed, as shown in Fig. 2. For the estimation of the mean amplitude distribution $P, 36$ phase bins were used. Then the MIs between each brain region and SCR were estimated, denoted by $\mathrm{MI}_{\text {prefront }}, \mathrm{MI}_{\text {central }}$, $\mathrm{MI}_{\text {temp }}, \mathrm{MI}_{\text {parietal }}, \mathrm{MI}_{\text {occip }}$. The final interdependence value per segment was estimated as the average interdependence between the EEG segments that emanate from the same brain region and the corresponding SCR segment, leading to 14 interdependence values per trial and brain region. An example MI time evolution for a trial and various brain regions is presented in Fig. 3. $\mathrm{MI}_{\text {temp }}$ seems to be larger than any other MI.The mean elapsed time for estimating an MI curve for 10 -sec windows with $60 \%$ overlap was 0.79 seconds (the algorithm was repeated 2500 times). The implementations were done on a laptop Duo Processor T7500 with 2048 MB RAM.

In order to compare the various MIs among all subjects, a statistical analysis was performed. In particular, the median of the MI values in each trial was extracted to represent the central tendency of the interdependencies in each brain region and trial. Then, multiple pairwise comparisons were carried out on the median values using a non-parametric bootstrap 


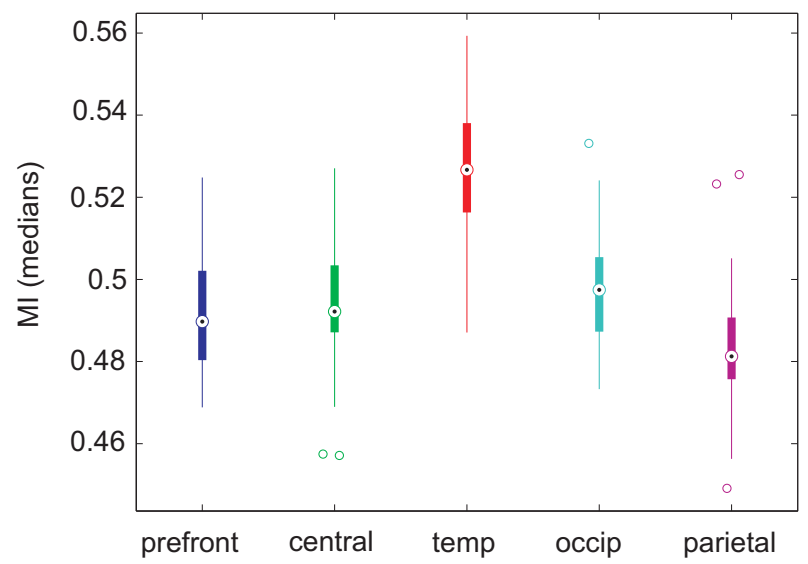

Fig. 4. Example of the medians of each MI for one subject. On each box, the central circle is the median, the edges of the box are the 25th and 75th percentiles, the whiskers extend to the most extreme data points not considered outliers, and outliers are plotted individually by circles.

hypothesis test [20] for each subject. Bonferroni's correction was then applied to the $p$-values to avoid multiple Type I errors. Finally a Stouffer's meta-analysis test provided the final $p$-value for each comparison. Stouffer's test combines the $p$ values of independent tests (different subjects) which share the same null hypothesis (MI in a brain region larger than $\mathrm{MI}$ in another). This test was selected because it outperforms other meta-analysis tests [21].

The results reveal indeed that $\mathrm{MI}_{t e m p}$ is significantly higher than the other MIs $(p<0.001)$. The order of the remaining MIs is the following; $\mathrm{MI}_{\text {occip }}>>\mathrm{MI}_{\text {central }}>>$ $\mathrm{MI}_{\text {parietal }}>>\mathrm{MI}_{\text {prefront }}$. The symbol $>>$ indicates that each MI is significantly larger with $p<0.01$. An example of MI median values for one subject is displayed in Fig. 4.

\section{B. Relation with the subjective ratings}

In the DEAP database subjects were asked to rate each music clip in terms of their self-assessed valence and arousal [19], [22]. In order to explore whether the relative relationship among the MIs changes with respect to the subjective ratings, the following analysis was performed. The self-assessed ratings were automatically clustered into six categories, namely high arousal (HA), low arousal (LA), neutral arousal (NA), high valence (HV), low valence (LV), and neutral valence (NV), using the K-means clustering algorithm. The statistical analysis presented in III-A was applied to each cluster, and the results revealed that the order of the MIs was always as in III-A independently of the cluster. Thus, the synchronization between temporal EEG and SCR remains the strongest independently of the emotion elicited by the music clip.

Moreover, in order to investigate the way each MI is related to the subjective ratings, a similar statistical analysis was applied to each MI for $\mathrm{HV}, \mathrm{LV}$, and NV music clips, as well for HA, LA, and NA music clips. The results revealed that the coupling between temporal/central EEG and SCR increases significantly $(p<0.01)$ for HA compared to NA and LA music clips (examples presented in Fig. 5), whereas

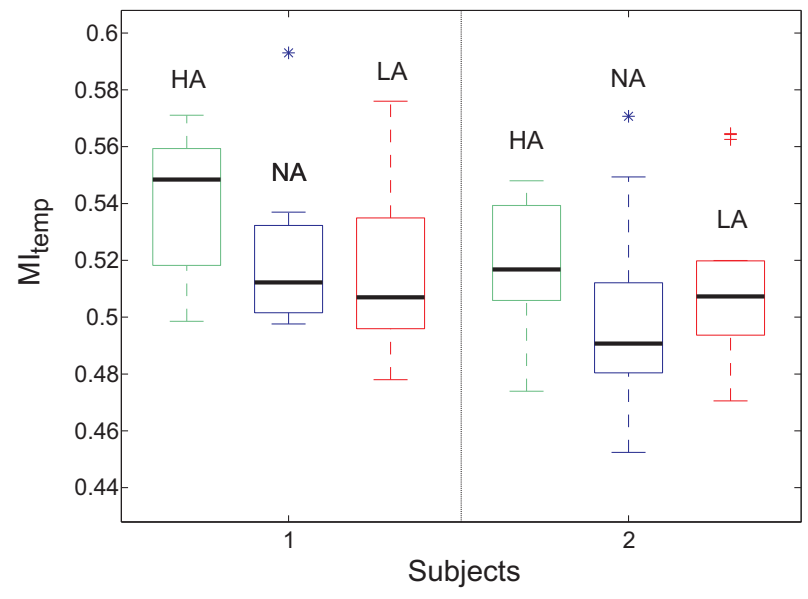

Fig. 5. MI temporal for high arousal (HA), neutral arousal (NA), and low arousal (LA) for two subjects. On each box, the central black line is the median, the edges of the box are the 25 th and 75 th percentiles, the whiskers extend to the most extreme data points not considered outliers, and outliers are plotted individually either by crosses, circles, or stars.

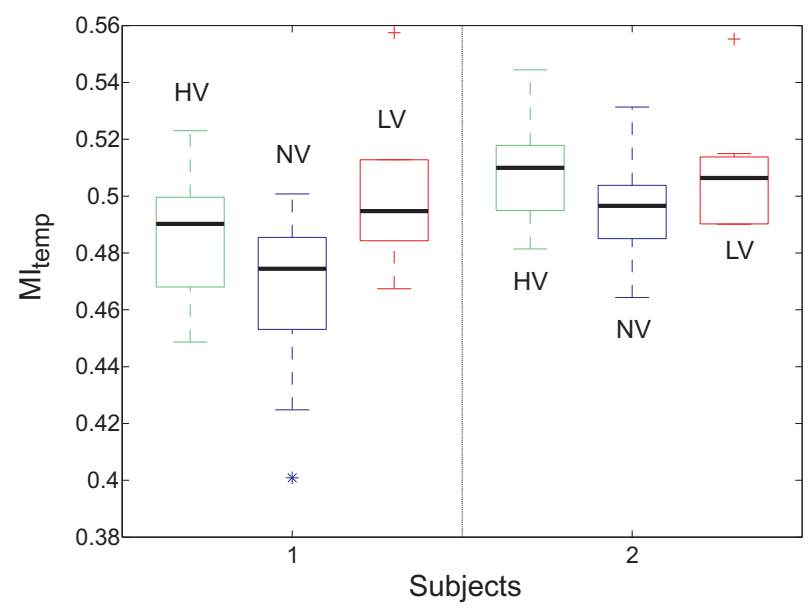

Fig. 6. MI temporal for high valence (HV), neutral valence (NV), and low valence $(\mathrm{LV})$ for two subjects. On each box, the central black line is the median, the edges of the box are the 25th and 75th percentiles, the whiskers extend to the most extreme data points not considered outliers, and outliers are plotted individually either by crosses, circles, or stars.

no significant differences were observed on temporal/central EEG and SCR coupling for NA and LA music clips. There were not any significant differences for the rest of the MIs regarding arousal. Moreover, temporal EEG and SCR coupling increases significantly $(p<0.01)$ for HV and for LV music clips compared to NV ones, whereas no difference is observed for $\mathrm{HV}$ and $\mathrm{LV}$ music clips, indicating that the synchronization between temporal EEG and SCR follows a U-shape with respect to valence. An example of such a synchronization is presented in Fig. 6 for two subjects.

\section{DISCUSSION}

The idea that SCR interacts with brain signals during emotional processes is also supported by neurophysiological studies. For instance, Damasio's brain-body theory [1] suggests that brain and body are not two individual entities, but 
that one influences the other, and their interactions play a crucial role in our emotional experiences and decision making. In particular, emotional stimuli seem to activate the amygdala, which in turn automatically activate networks that control the appearance of visceral responses (autonomic responses such as respiration, skin conductance and hormonal responses) [23]. Visceral responses send back messages to the brain [23], indicating that body dynamics activate brain dynamics, too. This theory of bodily feedback during emotional reactions was first supported by James [24] who argued, in a nutshell, that we do not cry because we are sad or run because we are afraid, but we are sad because we cry and afraid because we run. Moreover, it is also known that amygdala interacts with the early sensory cortices, such as occipitotemporal and insula, by sending messages to these cortices, enhancing the neural representations of biologically and emotionally significant stimuli [25].

In our experiment, the interactions of SCR and EEG signals increase significantly when the phase of SCR modulates the amplitude of the EEG signals of the temporal lobe, independently of the stimulus. The fact that the coupling between temporal EEG and SCR increases significantly for high-arousal music clips compared to low-arousal ones, as well as for high-valence and low-valence music clips compared to neutral ones, indicates that this coupling is strongest when strong emotions are elicited (neutral valence and low arousal indicate less strong emotions). This also supported by Scherer's theory on appraisals [13], according to which emotions exist as a result of synchronization or interaction of subsystems of the organism. The above finding also corroborates with [17], in which it was shown that phase synchronization of heart rate and respiration increases significantly for high-arousal images compared to neutral ones. Since the temporal lobe is associated with the auditory cortex, we might speculate that during music clips, music is a more emotionally significant stimulus than the video.

The above result also corroborates with another study [26] that revealed higher classification accuracy for valence and arousal based on EEG signals for audio and audiovisual stimuli than visual stimuli only. Also, in [27], the authors demonstrated enhanced emotional experience by adding music to pictures. Thus, apparently, music seems to be a more emotionally significant stimulus than the video. Though, it could be quite interesting to investigate whether similar interactions take place when subjects are watching films rather than music clips or whether in this case the occipital EEG signals are more coupled with visceral responses, indicating that the movie content is more important than the audio.

Finally, the occipital lobe is associated with the visual cortex, the central lobe with the somatosensory and motor cortex, and the parietal corresponds to the lobe in which the visual with the auditory cortex meet. Hence, it seems that the SCR triggers more the sensory cortices (auditory, visual, somatosensory, motor and audiovisual) than the prefrontal cortex, although the latter is highly associated with affect [6], [5]. A possible reason for this could be that, although associated with affect its role differs from the role of amygdala in affect. In fact, it is known that the orbitofrontal cortex (which is a part of the prefrontal cortex) is responsible for the rapid and flexible adjustment of emotional responses depending on the contingencies of the emotional stimuli. This is actually the reason why patients with frontal lobe damages have normal intelligence scores, interact in a normal way with the environment, but lack having "secondary" emotional responses and decision making abilities [1]. On the other hand, amygdala is crucial for acquisition and retention of emotional associations, thus, very much linked to Damasio's "primary" emotions [1], indicating that temporal EEG/SCR interactions are probably more linked to primary emotions than secondary ones.

Apart from the neuropsychological value of this study, the interactions between temporal EEG signals and SCR captured by the phase of SCR and amplitude of EEG signals, can have a great impact in affective computing. First, PAC captures the interactions very fast, so it could be easily used for realtime applications. In fact, physiological signals interactions in affective computing can be used as a means of identifying implicitly strong subjective emotional segments.In particular, this can be used to extract emotional highlights and discard the segments that could negatively affect the accuracy of the emotion recognition systems. Also, the interactions between temporal EEG and SCR indicate whether a stimulus elicits high or low arousal, as well as high/low or neutral valence. Thus, a music clip can be automatically depicted in the 2-D VA space, and clustered in terms of the strength of the emotion it elicits. Moreover, possible similarities or discrepancies among the various MIs can yield additional information about the way each person perceives each music clip. A possible relationship between the highest MI for a subject and a music clip can indicate whether this particular subject bases his/her ratings on the music or video content, as well as which part of his/her brain is activated. Finally, common patterns among subjects that arise from the drifts of the MIs might cluster the subjects based on their physiological signals interactions and pave the way for automatic user profiling.

\section{CONCLusions}

In this study we explored the phase-amplitude coupling between SCR and EEG signals of different brain regions while experiencing music clips. In particular, we investigated the way the instantaneous phase of SCR affects the amplitude of EEG signals of the prefrontal, central, temporal, parietal and occipital lobes. The results revealed that the SCR is more coupled with the EEG signals of the temporal lobe. Moreover, the coupling between temporal EEG and SCR increases with high- arousal music clips, as well as with high and lowvalence music clips, compared to neutral ones. These findings corroborate with other studies on synchronization phenomena among different organismic subsystems occuring when strong emotions are experienced. Since the temporal lobe is associated with the auditory cortex, the above findings indicate that music enhances the emotional experience more than the 
video content. Moreover, SCR seems more coupled with the sensory cortices (auditory, visual, somatosensory, motor and audiovisual) than with the prefrontal cortex. Since the sensory cortices are known to interact with amygdala which is responsible for primary emotions, the coupling between SCR and EEG is probably driven by primary emotional processes, instead of cognitive appraisal processes.

\section{ACKNOWLEDGMENT}

The research leading to these results has been performed in the frameworks of Swiss National Foundation for Scientific Research (FN 200020-132673-1), and the NCCR Interactive Multimodal Information Management (IM2).

\section{REFERENCES}

[1] A. Damasio, Descartes' error: Emotion, Reason and the Human Brain. Putnam New York, 1994.

[2] S. Kreibig, "Autonomic nervous system activity in emotion: A review," Biological psychology, vol. 84, no. 3, pp. 394-421, 2010.

[3] M. Dawson, A. Schell, and D. Filion, "The electrodermal system," Handbook of psychophysiology, vol. 2, pp. 200-223, 2000.

[4] Y. Nagai, H. Critchley, E. Featherstone, M. Trimble, and R. Dolan, "Activity in ventromedial prefrontal cortex covaries with sympathetic skin conductance level: a physiological account of a default mode of brain function," Neuroimage, vol. 22, no. 1, pp. 243-251, 2004.

[5] R. Davidson, "What does the prefrontal cortex do in affect: perspectives on frontal EEG asymmetry research," Biological psychology, vol. 67, no. 1, pp. 219-234, 2004.

[6] J. Coan and J. Allen, "Frontal EEG asymmetry as a moderator and mediator of emotion," Biological psychology, vol. 67, no. 1, pp. 7-50, 2004.

[7] Y. Lin, C. Wang, T. Jung, T. Wu, S. Jeng, J. Duann, and J. Chen, "EEGbased emotion recognition in music listening," Biomedical Engineering, IEEE Transactions on, vol. 57, no. 7, pp. 1798-1806, 2010.

[8] E. Kroupi, A. Yazdani, J.-M. Vesin, and T. Ebrahimi, "Multivariate spectral analysis for identifying the brain activations during olfactory perception," in Engineering in Medicine and Biology Society (EMBC), 2012 Annual International Conference of the IEEE. IEEE, 2012, pp. $6172-6175$.

[9] G. Chanel, C. Rebetez, M. Bétrancourt, and T. Pun, "Emotion assessment from physiological signals for adaptation of game difficulty," Systems, Man and Cybernetics, Part A: Systems and Humans, IEEE Transactions on, vol. 41, no. 6, pp. 1052-1063, 2011.

[10] M. Soleymani, M. Pantic, and T. Pun, "Multimodal emotion recognition in response to videos," Affective Computing, IEEE Transactions on, vol. 3, no. 2, pp. $211-223$, april-june 2012.

[11] R. Picard, E. Vyzas, and J. Healey, "Toward machine emotional intelligence: Analysis of affective physiological state," Pattern Analysis and Machine Intelligence, IEEE Transactions on, vol. 23, no. 10, pp. 11751191,2001

[12] S. Koelstra, A. Yazdani, M. Soleymani, C. Muehl, J.-S. Lee, A. Nijholt, T. Pun, T. Ebrahimi, and I. Patras, "Single trial classification of EEG and peripheral physiological signals for recognition of emotions induced by music videos," in Proceedings of the International Conference on Brain Informatics, 2010.

[13] K. Scherer, "Appraisal considered as a process of multilevel sequential checking," Appraisal processes in emotion: Theory, methods, research, vol. 92, pp. 120-128, 2001.

[14] H. Critchley, R. Melmed, E. Featherstone, C. Mathias, and R. Dolan, "Volitional control of autonomic arousal: a functional magnetic resonance study," Neuroimage, vol. 16, no. 4, pp. 909-919, 2002.

[15] P. Bob, "Hypnotic abreaction releases chaotic patterns of electrodermal activity during dissociation," Intl. Journal of Clinical and Experimental Hypnosis, vol. 55, no. 4, pp. 435-456, 2007.

[16] W. Pritchard and D. Duke, "Measuring chaos in the brain: a tutorial review of nonlinear dynamical EEG analysis," International Journal of Neuroscience, vol. 67, no. 1-4, pp. 31-80, 1992.
[17] G. Valenza, A. Lanata, and E. P. Scilingo, "Oscillations of heart rate and respiration synchronize during affective visual stimulation." Information Technology in Biomedicine, IEEE Transactions on, vol. 16, no. 4, pp. 683-690, 2012.

[18] A. B. Tort, R. Komorowski, H. Eichenbaum, and N. Kopell, "Measuring phase-amplitude coupling between neuronal oscillations of different frequencies," Journal of neurophysiology, vol. 104, no. 2, pp. 11951210, 2010.

[19] S. Koelstra, C. Muhl, M. Soleymani, J. Lee, A. Yazdani, T. Ebrahimi, T. Pun, A. Nijholt, and I. Patras, "DEAP: A database for emotion analysis; using physiological signals," Affective Computing, IEEE Transactions on, vol. 3, no. 1, pp. 18-31, 2012.

[20] B. Efron and R. J. Tibshirani, An Introduction to the Bootstrap. LinkNew York: Chapman \& Hall, 1993.

[21] M. Whitlock, "Combining probability from independent tests: the weighted z-method is superior to fisher's approach," Journal of evolutionary biology, vol. 18, no. 5, pp. 1368-1373, 2005.

[22] J. A. Russell, "A circumplex model of affect," Journal of Personality and Social Psychology, vol. 39, pp. 1161-1178, 1980.

[23] J. LeDoux, The emotional brain: The mysterious underpinnings of emotional life. Simon \& Schuster, 1998.

[24] W. James, The principles of psychology. Henry Holt and Co, 1890.

[25] J. Morris, K. Friston, C. Büchel, C. Frith, A. Young, A. Calder, and R. Dolan, "A neuromodulatory role for the human amygdala in processing emotional facial expressions." Brain, vol. 121, no. 1, pp. 4757, 1998.

[26] D. O. Bos, "EEG-based emotion recognition," The Influence of Visual and Auditory Stimuli, 2006.

[27] T. Baumgartner, M. Esslen, L. Jäncke et al., "From emotion perception to emotion experience: Emotions evoked by pictures and classical music," International Journal of Psychophysiology, vol. 60, no. 1, pp. 34-43, 2006. 